\title{
A BILATERAL SERIES INVOLVING BASIC HYPERGEOMETRIC FUNCTIONS
}

\author{
HJALMAR ROSENGREN
}

Dedicated to Mizan Rahman

\begin{abstract}
We prove a summation formula for a bilateral series whose terms are products of two basic hypergeometric functions. In special cases, series of this type arise as matrix elements of quantum group representations.
\end{abstract}

\section{INTRODUCTION}

The object of the present paper is to study bilateral series of a type that first appeared in the work of Koelink and Stokman [KS], in the context of harmonic analysis on the $\mathrm{SU}(1,1)$ quantum group. They needed to compute sums of the form

$$
\sum_{n=-\infty}^{\infty}{ }_{2} \phi_{1}\left(\begin{array}{c}
a, b \\
c
\end{array} ; q, x q^{n}\right){ }_{2} \phi_{1}\left(\begin{array}{c}
d, e \\
f
\end{array} ; q, y q^{n}\right) t^{n},
$$

which appeared as matrix elements of quantum $\mathrm{SU}(1,1)$ representations. Several special cases were considered, with different, very technical, proofs (some of these proofs were found by Mizan Rahman, see the appendix to [KS]).

In joint work with Koelink $\mathrm{KR}$, we gave an extension of the summation formulas from [KS, with a unified proof. Namely, we showed that under natural conditions of convergence and the assumption

$$
\text { abde }=c f, \quad f x=d e y
$$

(and thus also $c y=a b x$ ), the sum in (11) can be expressed as the sum of two ${ }_{8} W_{7}$ series, or, alternatively, as the sum of three balanced ${ }_{4} \phi_{3}$ series. From the quantum algebraic viewpoint, which was only briefly mentioned in $[\mathrm{KR}$, this computes a general class of matrix elements for the strange, the complementary and the principal unitary series of quantum $\mathrm{SU}(1,1)$. The proof in $[\mathrm{KR}]$ is similar to, though not an extension of, one of Rahman's proofs in $[\mathrm{KS}$. In particular, it is quite technical and involves rather non-obvious applications of $q$-series transformations.

In a related paper [S], Stokman considered matrix elements for the principal unitary series and gave a very simple proof for their expression as ${ }_{8} W_{7}$ series. The idea is simple but powerful: the representations are realized on $\mathrm{L}^{2}(\mathbb{T})$, where one wants to compute the scalar product of two particular functions. Expanding both functions as Fourier series gives a special case of the sum (1). Stokman's observation, which is our starting point, is that the integral defining the scalar product can be computed very easily using residue calculus. 
Our aim is to use Stokman's method not only to give a simple proof of the summation formula in [KR], but also to give a far-reaching extension of this identity.

Dedication: This paper would not have been written without the pioneering contributions of Mizan Rahman. I'm happy to know him, not only through his work as a master of identities, but also as a genuinely kind and helpful person. It is a pleasure to dedicate this paper to him as a small token of my appreciation.

\section{A Bilateral summation}

We will now state the main result of the paper. Throughout, we use the standard notation of GR. The base $q$ is assumed to satisfy $0<|q|<1$. We denote by ${ }_{r+1} \phi_{r}$ not only the convergent basic hypergeometric series, but also its analytic continuation to $\mathbb{C} \backslash \mathbb{R}_{\geq 1}$; cf. $[\mathrm{GR}, \S 4.5]$.

Theorem 2.1. Let $x, y \in \mathbb{C} \backslash \mathbb{R}_{>0}$, and let the other parameters below satisfy

$$
\begin{gathered}
\max _{i j}\left(\left|a_{i} c_{j}\right|\right)<|t|<1, \\
\left|q y b_{1} \cdots b_{k}\right|<\left|x a_{1} \cdots a_{k+1}\right| .
\end{gathered}
$$

Then the following identity holds:

$$
\sum_{n=-\infty}^{\infty}{ }_{k+1} \phi_{k}\left(\begin{array}{c}
a_{1}, \ldots, a_{k+1} \\
b_{1}, \ldots, b_{k}
\end{array} ; q, x q^{n}\right){ }_{l+1} \phi_{l}\left(\begin{array}{c}
c_{1}, \ldots, c_{l+1} \\
d_{1}, \ldots, d_{l}
\end{array} ; q, y q^{n}\right) t^{n}
$$

$$
=\frac{\left(q, x t, q / x t, a_{1}, \ldots, a_{k+1}, b_{1} / t, \ldots, b_{k} / t ; q\right)_{\infty}}{\left(t, x, q / x, a_{1} / t, \ldots, a_{k+1} / t, b_{1}, \ldots, b_{k} ; q\right)_{\infty}}
$$

$$
\times_{k+l+2} \phi_{k+l+1}\left(\begin{array}{c}
t, q t / b_{1}, \ldots, q t / b_{k}, c_{1}, \ldots, c_{l+1} \\
q t / a_{1}, \ldots, q t / a_{k+1}, d_{1}, \ldots, d_{l}
\end{array} ; q, \frac{q y b_{1} \cdots b_{k}}{x a_{1} \cdots a_{k+1}}\right)
$$

$$
+\frac{\left(q, a_{1} x, q / a_{1} x, y t / a_{1}, q a_{1} / y t, a_{2}, \ldots, a_{k+1}, b_{1} / a_{1}, \ldots, b_{k} / a_{1} ; q\right)_{\infty}}{\left(x, q / x, y, q / y, t / a_{1}, a_{2} / a_{1}, \ldots, a_{k+1} / a_{1}, b_{1}, \ldots, b_{k} ; q\right)_{\infty}}
$$

$$
\times \frac{\left(c_{1}, \ldots, c_{l+1}, a_{1} d_{1} / t, \ldots, a_{1} d_{l} / t ; q\right)_{\infty}}{\left(a_{1} c_{1} / t, \ldots, a_{1} c_{l+1} / t, d_{1}, \ldots, d_{l} ; q\right)_{\infty}}
$$

$$
\times_{k+l+2} \phi_{k+l+1}\left(\begin{array}{c}
a_{1}, q a_{1} / b_{1}, \ldots, q a_{1} / b_{k}, a_{1} c_{1} / t, \ldots, a_{1} c_{l+1} / t \\
q a_{1} / t, q a_{1} / a_{2}, \ldots, q a_{1} / a_{k+1}, a_{1} d_{1} / t, \ldots, a_{1} d_{l} / t
\end{array} ; q, \frac{q y b_{1} \cdots b_{k}}{x a_{1} \cdots a_{k+1}}\right)
$$

$+\operatorname{idem}\left(a_{1} ; a_{2}, \ldots, a_{k+1}\right)$.

Here, idem $\left(a_{1} ; a_{2}, \ldots, a_{k+1}\right)$ denotes the sum of the $k$ terms obtained by interchanging $a_{1}$ with each of $a_{2}, \ldots, a_{k+1}$. As is customary, we implicitly assume that the parameters are such that one never divides by zero. Note also that interchanging the roles of ${ }_{k+1} \phi_{k}$ and ${ }_{l+1} \phi_{l}$ gives an alternative expression for the series, which is valid when (4) is replaced by the condition $\left|q x d_{1} \cdots d_{l}\right|<\left|y c_{1} \cdots c_{l+1}\right|$. These two expressions are related by [GR, (4.10.10)].

In $[\mathrm{KR}]$, we computed the sum when $k=l=1$ and, crucially to the methods used there, (2) holds. The latter condition means exactly that the three ${ }_{4} \phi_{3}$ series on the right-hand side of (5) are balanced. In this case Theorem 2.1 reduces to [KR, 
(3.13)], rather than the alternative expression as a sum of two ${ }_{8} W_{7}$ series given in [KR, Proposition 3.1].

Our main tool is the following lemma from $[\mathrm{KR}$, where it was used only in the case $k=5$. Here we need the general case.

Lemma 2.2. For

$$
\max _{i}\left(\left|a_{i}\right|\right)<|t|<1
$$

and $x \in \mathbb{C} \backslash \mathbb{R}_{>0}$ one has

$$
\begin{aligned}
\sum_{n=-\infty}^{\infty}{ }_{k+1} \phi_{k}\left(\begin{array}{c}
a_{1}, \ldots, a_{k+1} ; q, x q^{n} \\
b_{1}, \ldots, b_{k}
\end{array}\right) & t^{n} \\
& =\frac{\left(q, a_{1}, \ldots, a_{k+1}, x t, q / x t, b_{1} / t, \ldots, b_{k} / t ; q\right)_{\infty}}{\left(x, q / x, b_{1}, \ldots, b_{k}, t, a_{1} / t, \ldots, a_{k+1} / t ; q\right)_{\infty}} .
\end{aligned}
$$

In $[\mathrm{KR}$, this was proved using the explicit formula for analytic continuation of ${ }_{k+1} \phi_{k}$ series. It is possible to give a much simpler proof using integral representations. Namely, the coefficient of $t^{n}$ in the Laurent expansion of the right-hand side in the annulus ([6) is given by

$$
\frac{\left(q, a_{1}, \ldots, a_{k+1} ; q\right)_{\infty}}{\left(x, q / x, b_{1}, \ldots, b_{k} ; q\right)_{\infty}} \frac{1}{2 \pi i} \int \frac{\left(x t, q / x t, b_{1} / t, \ldots, b_{k} / t ; q\right)_{\infty}}{\left(t, a_{1} / t, \ldots, a_{k+1} / t ; q\right)_{\infty}} t^{-n-1} d t
$$

where the integral is over a positively oriented contour encircling the origin inside the annulus. This is an integral of the form GR, (4.9.4)], which is computed there using residue calculus. If $\left|x q^{n}\right|<1$, its value is given by [GR, (4.10.9)] as the ${ }_{k+1} \phi_{k}$ series in (77). Since (8) is analytic in $x$, this also holds for $\left|x q^{n}\right| \geq 1$ in the sense of analytic continuation.

Remark 2.3. More generally, [GR, (4.10.9)] may be used to express the Laurent coefficients of

$$
\prod_{i=1}^{m} \frac{\left(\alpha_{i} t ; q\right)_{\infty}}{\left(\gamma_{i} t ; q\right)_{\infty}} \prod_{i=1}^{n} \frac{\left(\beta_{i} / t ; q\right)_{\infty}}{\left(\delta_{i} / t ; q\right)_{\infty}}
$$

in the annulus

$$
\max _{i}\left|\delta_{i}\right|<|t|<\min _{i}\left(1 /\left|\gamma_{i}\right|\right)
$$

as sums of analytically continued basic hypergeometric series.

Proof of Theorem 2.1. Similarly as in [KR, it is easy to check that (3) is the natural condition for absolute convergence of the left-hand side of (5). We first rewrite this series as an integral. For this we make the preliminary assumption that $t$ is real with

$$
\max _{i j}\left(\left|a_{i}\right|,\left|c_{j}\right|\right)<t^{1 / 2}<1
$$

Writing $f_{k}(t ; a, b, x)$ for either side of (17), we consider the integral

$$
\frac{1}{2 \pi i} \int_{|z|=t^{1 / 2}} f_{k}(z ; a, b, x) f_{l}(\bar{z} ; c, d, y) \frac{d z}{z} \text {. }
$$


Using the expression on the left-hand side of (7) together with orthogonality of the monomials, we find that it equals the sum in (5).

To compute the integral, we plug in the expressions from the right-hand side of (77). The integrand is then of the form (9), with $\alpha=\left(x, q / y t, d_{1} / t, \ldots, d_{l} / t\right)$, $\beta=\left(q / x, y t, b_{1}, \ldots, b_{k}\right), \gamma=\left(1, c_{1} / t, \ldots, c_{l+1} / t\right), \delta=\left(t, a_{1}, \ldots, a_{k+1}\right)$. Note that (10) and (11) are equivalent. Thus, we again have an integral of the form GR, (4.9.4)]. The condition (4) is precisely [GR, (4.10.2)], which ensures that the singularity at $z=0$ does not contribute to the integral. The value of the integral is then given by GR, (4.10.8)] as a sum of $k+2$ terms, each being a ${ }_{k+l+4} \phi_{k+l+3}$ series. However, because of the condition $\alpha_{1} \beta_{1}=\alpha_{2} \beta_{2}=q$, they all reduce to type ${ }_{k+l+2} \phi_{k+l+1}$. This proves Theorem [2.1] under the assumption (11). By analytic continuation in $t$, this may be replaced with the weaker condition (3).

Remark 2.4. Using instead of Lemma 2.2 the Laurent expansion of the general product (9) gives a generalization of Theorem 2.1. where the two basic hypergeometric series on the left-hand side of (5) are replaced by finite sums of such series.

In the case $l=0$, one may use the $q$-binomial theorem to sum the series ${ }_{1} \phi_{0}$ in Theorem 2.1. The condition $y \in \mathbb{C} \backslash \mathbb{R}_{>0}$ is then superfluous. We find this special case interesting enough to write out explicitly. Compared to Theorem 2.1 we have made the change of variables $y \mapsto c, c_{1} \mapsto d / c$.

Corollary 2.5. Let $x \in \mathbb{C} \backslash \mathbb{R}_{>0}$, and assume that

$$
\max _{i}\left(\left|a_{i} d / c\right|\right)<|t|<1, \quad\left|q c b_{1} \cdots b_{k}\right|<\left|x a_{1} \cdots a_{k+1}\right|
$$

Then the following identity holds:

$$
\begin{aligned}
\sum_{n=-\infty}^{\infty} \frac{(c)_{n}}{(d)_{n}}{ }_{k+1} & \phi_{k}\left(\begin{array}{c}
a_{1}, \ldots, a_{k+1} \\
b_{1}, \ldots, b_{k}
\end{array} ;, x q^{n}\right) t^{n} \\
= & \frac{\left(q, x t, q / x t, c, a_{1}, \ldots, a_{k+1}, b_{1} / t, \ldots, b_{k} / t ; q\right)_{\infty}}{\left(t, x, q / x, d, a_{1} / t, \ldots, a_{k+1} / t, b_{1}, \ldots, b_{k} ; q\right)_{\infty}} \\
& \quad \times_{k+2} \phi_{k+1}\left(\begin{array}{c}
t, d / c, q t / b_{1}, \ldots, q t / b_{k} \\
q t / a_{1}, \ldots, q t / a_{k+1}
\end{array} ; q, \frac{q c b_{1} \cdots b_{k}}{x a_{1} \cdots a_{k+1}}\right) \\
+ & \frac{\left(q, d / c, a_{1} x, q / a_{1} x, c t / a_{1}, q a_{1} / c t, a_{2}, \ldots, a_{k+1}, b_{1} / a_{1}, \ldots, b_{k} / a_{1} ; q\right)_{\infty}}{\left(x, q / x, q / c, d, a_{1} d / c t, t / a_{1}, a_{2} / a_{1}, \ldots, a_{k+1} / a_{1}, b_{1}, \ldots, b_{k} ; q\right)_{\infty}} \\
& \quad \times_{k+2} \phi_{k+1}\left(\begin{array}{c}
a_{1}, a_{1} d / c t, q a_{1} / b_{1}, \ldots, q a_{1} / b_{k} \\
q a_{1} / t, q a_{1} / a_{2}, \ldots, q a_{1} / a_{k+1}
\end{array} ;, \frac{q c b_{1} \cdots b_{k}}{x a_{1} \cdots a_{k+1}}\right) \\
+ & \operatorname{idem}\left(a_{1} ; a_{2}, \ldots, a_{k+1}\right) .
\end{aligned}
$$

Note that in the case $c=d$, the first ${ }_{k+2} \phi_{k+1}$ on the right-hand side reduces to 1 and the remaining terms to 0 . Thus, we recover Lemma 2.2. We also remark that if we choose $k=0$ in Corollary 2.5 and replace $x \mapsto a, a_{1} \mapsto b / a$, we obtain the 
transformation formula

$$
\begin{aligned}
{ }_{2} \psi_{2}\left(\begin{array}{c}
a, c \\
b, d
\end{array} ; q, t\right)= & \frac{(q, b / a, c, a t, q / a t ; q)_{\infty}}{(q / a, b, d, t, b / a t ; q)_{\infty}} \phi_{1}\left(\begin{array}{c}
t, d / c \\
a q t / b
\end{array} ; q, \frac{q c}{b}\right) \\
& +\frac{(q, q / b, d / c, a c t / b, q b / a c t ; q)_{\infty}}{(q / a, q / c, d, a t / b, b d / a c t ; q)_{\infty}}{ }_{2} \phi_{1}\left(\begin{array}{c}
b / a, b d / a c t \\
q b / a t
\end{array} ; q, \frac{q c}{b}\right) .
\end{aligned}
$$

This identity is also obtained by choosing $r=2, c_{1}=q a_{2}, c_{2}=b_{2}$ in [GR, (5.4.3)], and then applying [GR, (III.1)] to both ${ }_{2} \phi_{1}$ series on the right-hand side.

\section{REFERENCES}

[GR] G. Gasper and M. Rahman, Basic Hypergeometric Series, Cambridge University Press, Cambridge, 1990.

[KR] E. Koelink and H. Rosengren, Transmutation kernels for the little q-Jacobi function transform, Rocky Mountain J. Math. 32 (2002), 703-738.

[KS] E. Koelink and J. V. Stokman, with an appendix by M. Rahman, Fourier transforms on the quantum SU $(1,1)$ group, Publ. Res. Inst. Math. Sci. 37 (2001), 621-715.

[S] J. V. Stokman, Askey-Wilson functions and quantum groups, preprint, 2003, math.QA/0301330

Department of Mathematics, Chalmers University of Technology and Göteborg University, SE-412 96 Göteborg, Sweden

E-mail address: hjalmar@math.chalmers.se 